\title{
AN OPERATOR AIDING SYSTEM FOR COMPACTORS
}

\author{
Michel FROUMENTIN * and François PEYRET** \\ * Road Test Center - Le Grand Quevilly - FRANCE \\ ** Public Works Research Institute - Nantes - FRANCE
}

\section{SUMMARY}

This paper first describes the prototype operator aiding system called MACC, developed and realized by the "Road Test Center" in cooperation with the "Public Works Research institute". This on-board system displays a real-time two-dimensional colored cartography of the compaction pattern of the work site to inform the compactor operator about the level of energy already spent on the considered compaction area. The system can additionally provide the deferred data recorded for the control of the quality. Some results, achieved in actual field conditions, are then presented and the major developments of the system to contemplate in order to manufacture a standard device are finally discussed.

\section{INTRODUCTION}

In road construction, the compaction of embankments and pavement bases must be carried out keeping a uniform distribution of the compaction energy over the whole site. In order to obtain the required level of quality, the compactor operator needs to check that the number of passes is in accordance with the stipulated value for all points of the area to be compacted. This value is previously determined according to the type of work to be carried out.

The main difficulty met by the compactor operator is to post the number of passes actually performed on each point while managing cross-section changes of lanes on the roadway to be compacted.

Without any means and tools at his disposal to help him memorize his route, an operator, even an experienced one, which, what is more, must be vigilant for obvious safety reasons, quickly loses track of the work he has already done. 
The MACC operating aiding system has been designed to help the operator, to reduce the difficulty of his task and to increase the level of quality of the achieved compaction.

The device consists of an on-board computer, a man-machine interface and a positioning system essential to localize the compactor on the work site map. The entire device was designed with a view to simplifying the intervention of the operator as much as one can. The information is brought to the operator by a real-time two-dimensional map of the area to be compacted, which shows also the current position of the compactor.

The map is drawn with three levels of colour related to the various percentages of the current compaction energy level versus the required energy. The elementary mesh of the map is $1 \mathrm{~m}$ in the longitudinal section and $0.25 \mathrm{~m}$ in the cross-section.

During field tests, a standard positioning system, CAPSY ${ }^{\mathrm{TM}}$, has been used for the continuous tracking of the compactor position in the X, Y plane. CAPSY ${ }^{\mathrm{TM}}$ (Computer Aided Positioning SYstem) is manufactured by Spectra-Physics [1].

The principle of the MACC system, however, is independent from the positioning system and will enable to use a GPS system (Global Positioning System) in a close future.

All pieces of information about the map (number of passes performed on each elementary mesh) are stored in the on-board computer and transferred, when the work is completed, on a PC where they are post-processed by a special piece of software, which provides a statistical and cartographic treatment of the data.

The operational prototype discussed here should provide the information necessary to the design and development of a standard system fitting any type of compactor.

\section{DESCRIPTION OF THE MACC SYSTEM}

MACC consists of three on-board physical units loaded in a compactor (Figure 1): An on-board computer, a man-machine interface and a positioning sensor.

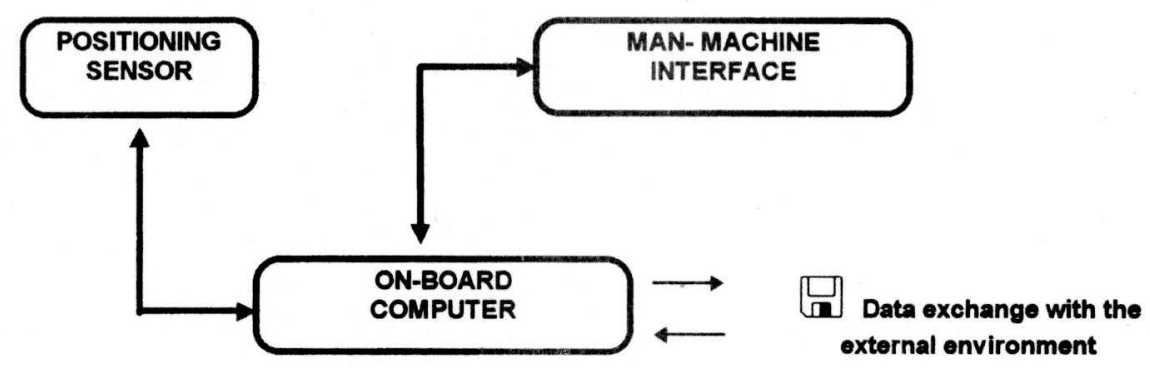

Figure 1: Architecture of the MACC system 
The system has been developed from an on-board standard PC. The choice was guided by the operating characteristics with regard to the environmental constraints. The PC is connected to a man-machine interface specially designed for this application. This unit, which is composed of a graphic screen equipped with a tactile sheet, comes as a dashboard independent from the computer.

The positioning sensor allows to located precisely the compactor on the work site map. The data link between the computer and the sensor is of the type RS232. Uncoupling the positioning system of the MACC system enables to use different sensors available on the market (GPS, optics, etc.). The CAPSY ${ }^{\mathrm{TM}}$ system, used with MACC during the tests, determines the position of a point by triangulation with the help of a rotating laser. During one rotation, the laser detects passive targets (indicated by a bar code mark) and measures the inter-beacon angles. When first knowing the position of the targets in a definite reference plane, the system is able to calculate, in the same reference plane, the position of the rotating laser provided that at least three targets are visible.

Thanks to this system coupled with MACC, the position in the $\mathrm{X}, \mathrm{Y}$ plan is obtained with an accuracy of approximately $+/-5 \mathrm{~cm}$ with an acquisition frequency of $4 \mathrm{~Hz}$.

Figure 2 shows the delimitation of the input and output of the MACC system.

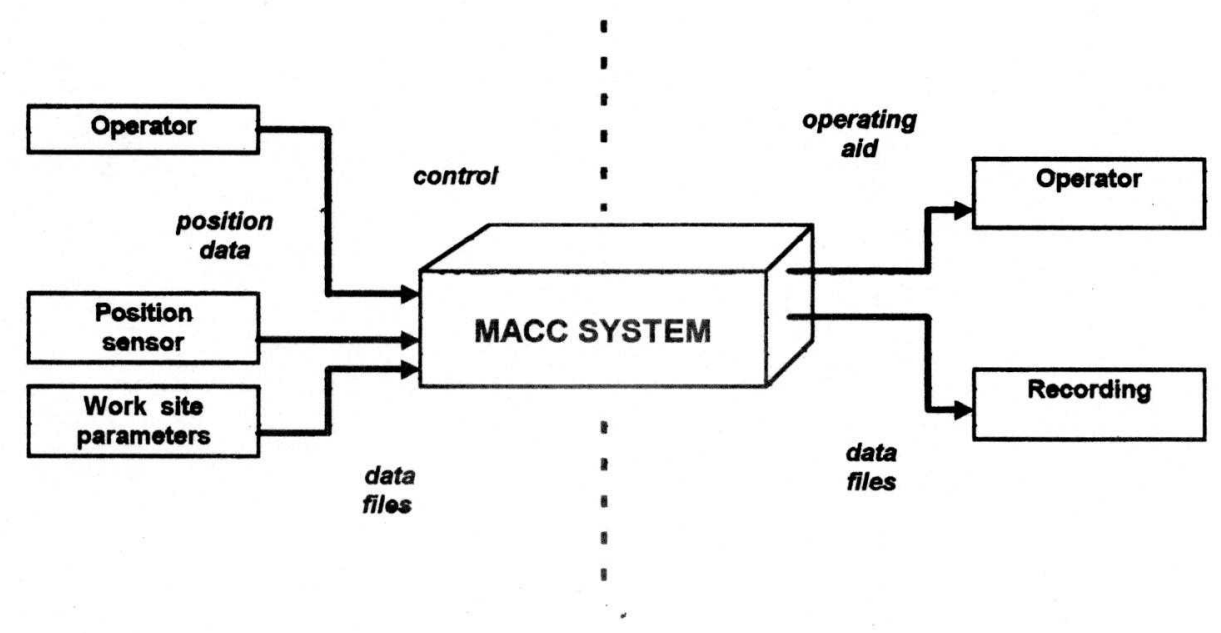

Figure 2: Input and output of the MACC system

During the compaction phase, the screen displays a schematic view of the work site over a finite length, which can be parameterized in the form of a two-dimensional map. In order to complete this representation, the MACC system requires to know all the parameters defining the work site. These parameters are relative to the geometry and topography of the work site in progress, on the one hand, and to the compaction practical conditions defined by the considered translation speed of the compactor and the number of passes to complete for each point, on the other hand.

Figure 3 illustrates the two different compaction phases, which are chronologically considered. 


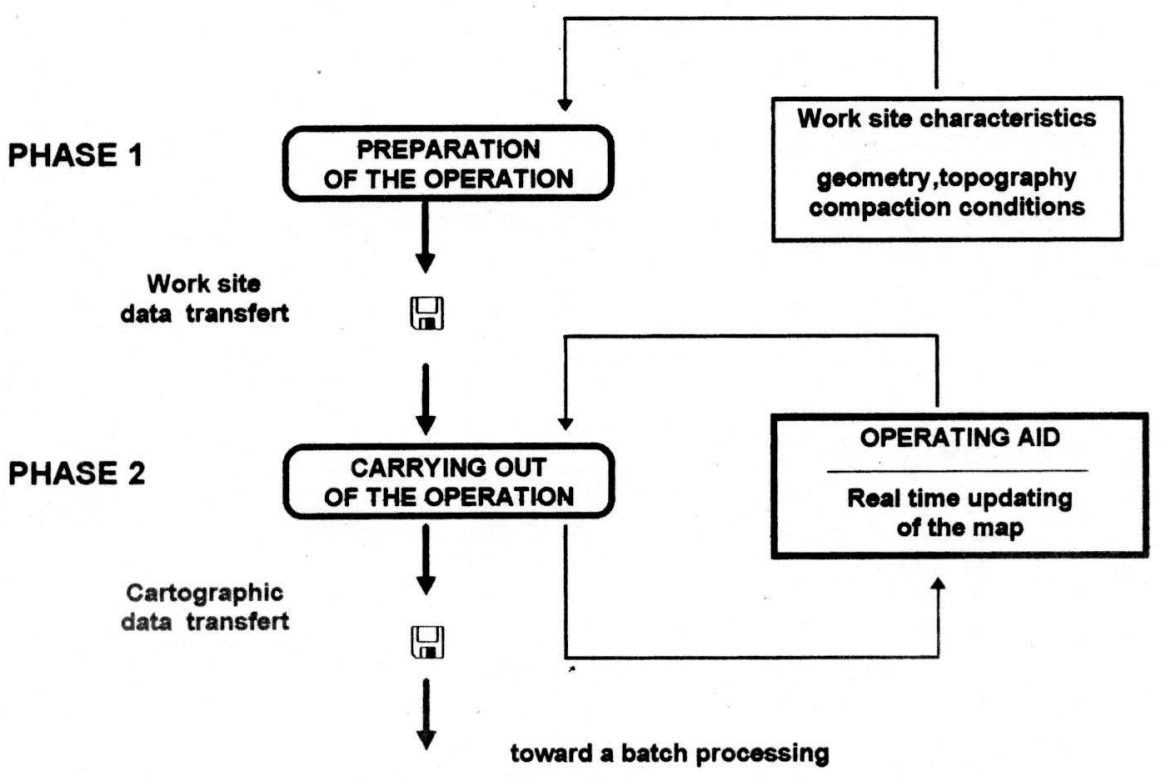

Figure 3: The two utilization phases of the MACC system

\section{Phase 1: "Preparation of the operation"}

In order to obtain the desired cartographic representation, the actual work site must be first modeled in the form of a rectangle composed of elementary rectangles (the meshes). This stage is completed thanks to a piece of software creating, from the work site elements (plans, CAD files, etc.) data files, which are directly usable by the MACC system. These files are relative to the geometry and the topography of the work site, to the cartography (work site mesh) and to the compaction conditions (translation speed and number of passes to complete).

For the MACC system, the work site is defined by all the axis profile points ( $\mathrm{Pi}$ ). A section (area separating two consecutive profile points) is materialized by a rectangle whereas, in fact, this section is defined by either a segment of a straight line, or an arc of a circle, or a portion of a clothoid.

The files generated by the software associated to phase 1 can be copied on diskettes.

\section{Phase 2: "Carrying out of the operation"}

During this phase, which reveals the true interest of the MACC system, the data created during phase 1 are loaded in the on-board computer, the operating aiding system is implemented by displaying the useful parameters on the dashboard and, lastly, the real-time cartographic file is updated during the progress of the operation. Thanks to this real-time storage, the operator keeps track of the work already completed. At the conclusion of a test sequence, a full day of compaction for instance, the software associated to phase 2 also allows to transfer the undated data on diskettes for possible batch processing. 


\section{DESCRIPTION OF THE OPERATING AID FUNCTION}

When all setup procedures have been performed (preparation, transfer of data files, starting of the MACC system), the operator is then able to use the operating aid equipment. With the tactile screen, he selects the profile, from which he is going to carry out the compaction operation, in the list of profile numbers making up the whole work site.

After validation, the system displays the operating aiding screen (Figure 4).

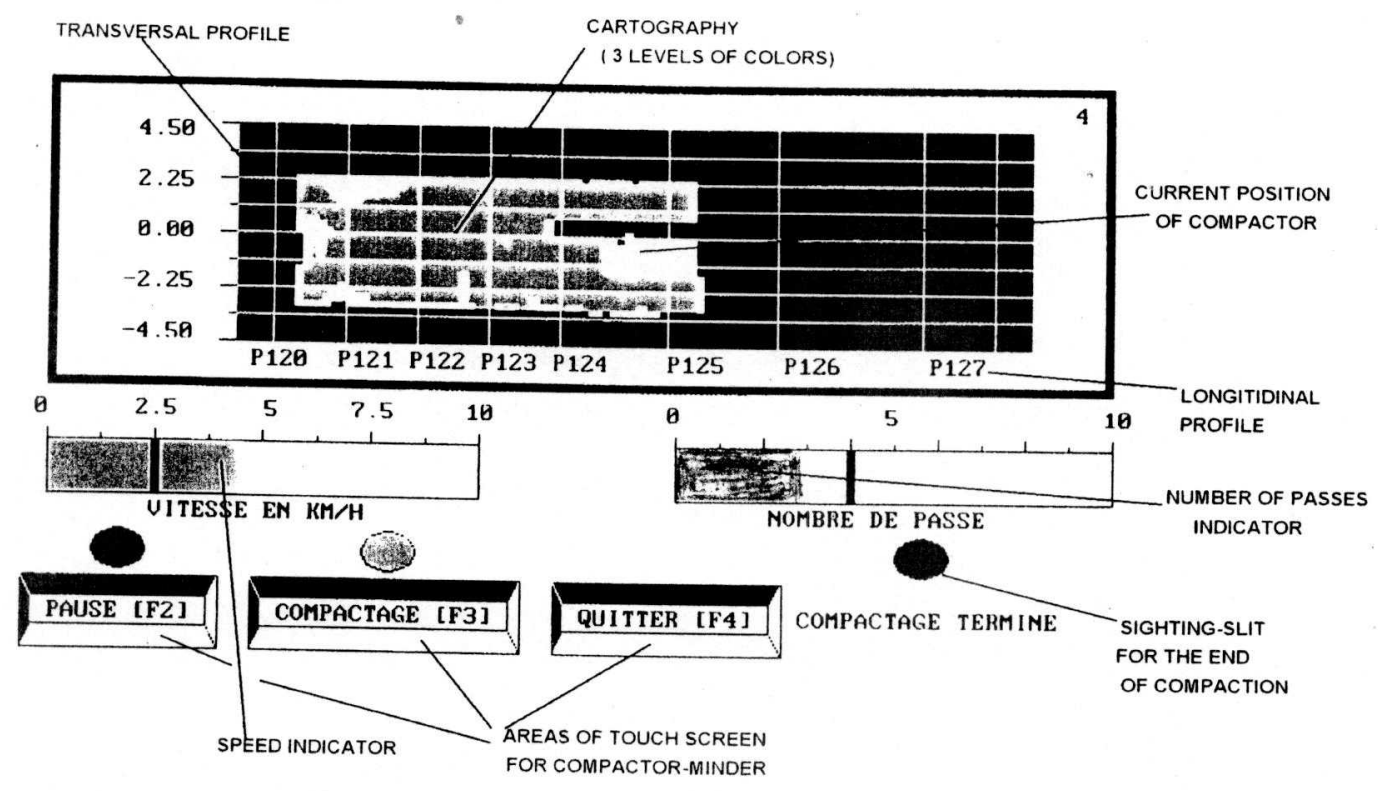

Figure 4: copy of the operating aiding screen

The schematic representation of the work site appears in the form of a rectangle. On the $X$ axis, longitudinal profile numbers are represented from the profile number previously selected by the operator. The longitudinal profile scale is respected and generally corresponds to a work site length of 100 meters. This value can be parameterized in the software within the range $25-400$ meters. Each cross-section profile is materialized by a vertical segment of a straight line on the whole width of the work site. The representation of the work site is then broken into a succession of rectangles corresponding to the different sections.

On the screen symbolizing the plane view of the work site, the three following representations can be distinguished:

\section{The current position of the compactor:}

The positioning sensor linked to the MACC system provides the position of the compactor. Once processed by the calculation algorithms, the position of the compactor in the reference 
screen described above, can be inferred. The algorithms also provide the value separating the longitudinal axis of the compactor from the work site axis.

Besides, since the width of the compactor is also known, the software is able to compute the current position of the compactor on the work site, materialized by a rectangle. A specific color represents the rectangle on the screen. The short side of the rectangle corresponds to an elementary mesh in the longitudinal profile $(1 \mathrm{~m})$, whereas the long side stands for the meshes covered by the compactor in the cross-section.

The display, which symbolizes the compactor, is carried out on a whole number of meshes. By definition, a mesh is covered by the compactor if, in the cross-section, the width of the compactor covers at least $75 \%$ of the mesh width. This rule is also applied to the end meshes located on the left and right sides of the compactor.

The screen also displays the longitudinal axis of the compactor.

Whenever the position sensor delivers new data, they are computed by the system, which provides the new precise position of the compactor. When the compactor crosses the limits of the site portion displayed on the screen, the rectangle disappears.

\section{Axises of the compaction strips:}

In order to do his work according to the rule book, the operator must perform the compaction strip after strip shifting in the cross-section whenever he needs to move from one strip to the other (one strip corresponds to the compactor width). The number of strips, the overlap between the strips and the position of each strip are defined and known beforehand and stored in the system. The MACC system displays on the screen the axis of each compaction strip to be followed by the operator in the form of straight lines over the full length of the longitudinal profile.

So as to make his work easier, the operator has the longitudinal axis of his compactor, displayed on the screen, correspond to each compaction strip. In that way, he makes sure that he respects the covering pattern and performs a homogeneous distribution of the compaction energy in the cross-section of the work site. He optimized the output of his compactor by avoiding extensive strip overlaps.

\section{Cartography:}

The rule book also obliges the operator to make sure that he performs the stipulated number of passes at all points of the work site. The operating aiding system stores the number of passes carried out by the compactor on each elementary mesh.

Furthermore, three thresholds of compaction energy are defined according to the ratio of the number of passes carried out by the compactor versus the number of stipulated passes (expressed in \%).

The thresholds are defined by the following values: (threshold 1 is reached if $\mathrm{R}<50 \%$ ), (threshold 2 is reached if $50 \%<\mathrm{R}<100 \%$ ) and (threshold 3 is reached if $\mathrm{R} \geq 100 \%$ ).

Compaction is assessed standard if threshold 3 is reached.

As the number of passes over each mesh is continuously known, the ratio $\mathrm{R}$ can be calculated for each mesh and the threshold reached on each elementary mesh is displayed with three different colors. Each threshold is symbolized by one of the color. 
The real-time management of the meshes is performed according to the following stages:

- At the time of the first display, all the meshes of the considered area of the work site are represented on the operating aiding screen with the color corresponding to the compaction threshold reached on them.

- The current position of the compactor is also displayed.

- Knowing the precise position of the compactor, the meshes covered by the compactor are identified and incremented if the compactor is performing a translation movement (speed $>0)$.

The possibility that the compactor may cross the limits of the work site on the left or right sides, when compacting on the edges, is considered by the calculation algorithms.

When an increment cycle is over, only the meshes, which have been modified, are displayed again on the screen with a possible change of their color according to the threshold reached. The partial display avoids a full screen erase, followed by a full screen display.

In the lower part of the screen, two graph bars display some information. The graph bar on the left of the screen provides the instantaneous translation speed of the compactor. The information of the speed vector is calculated from the data supplied by the position sensor between two successive acquisitions.

On the graph bar, the translation speed that the operator is required to maintain within a certain interval of tolerance, is indicated by a vertical index. The operator is required to control his speed by roughly maintaining the level of the graph bar with the mark corresponding to the ordered speed.

The right graph bar provides the number of passes already performed by the compactor on the elementary mesh corresponding to the current position of the compactor. Whenever the mesh changes, the value displayed on the graph bar is updated. Thanks to this system, the operator can obtain a more accurate view of the global display brought to him in the form of a map. This readout gives him precisely the number of passes performed where he stands with the compactor and not a global representation, with only three levels of color, of the area he is compacting anymore.

Similarly to the translation speed display, the number of ordered passes (number of passes to perform at each point) is materialized by a vertical index.

Finally, a light, called "COMPACTION OVER", appears under this graph bar. If the compactor stands on a mesh where the number of passes performed is plainly lower to the number of passes ordered, then the light is off. The light is on, indicating that compaction is over, if the compactor stands on a mesh where the number of passes performed is equal or higher than the ordered number of passes.

This information, which adds to the one provided by the cartography, allows to attract the operator's attention pointing out that the number of passes is reached and that he can move to another compaction strip for example. 


\section{FIELD TESTS}

The MACC prototype has been tested on a road construction site implementing a bituminous-bound graded aggregate layer. The test was carried out in collaboration with the French road builder, Cochery Bourdin Chaussé, last August 1995 on the national road RN2 (Lévignen loop). The equipment used is a tandem compactor (Dynapac CC501). Before the compaction test starts on the bituminous-bound graded aggregate layer, several sections, of a linear length of $100 \mathrm{~m}$, have been prepared to place the beacons of the CAPSY ${ }^{\mathrm{TM}}$ positioning system and to plot their precise coordinates.

Figure 5 shows the overall view of the site and of the compactor equipped with the rotating laser placed on the roof of the cab. Two of the CAPSY ${ }^{\mathrm{TM}}$ beacons are also visible in the background (the poles located on the top of the embankment).

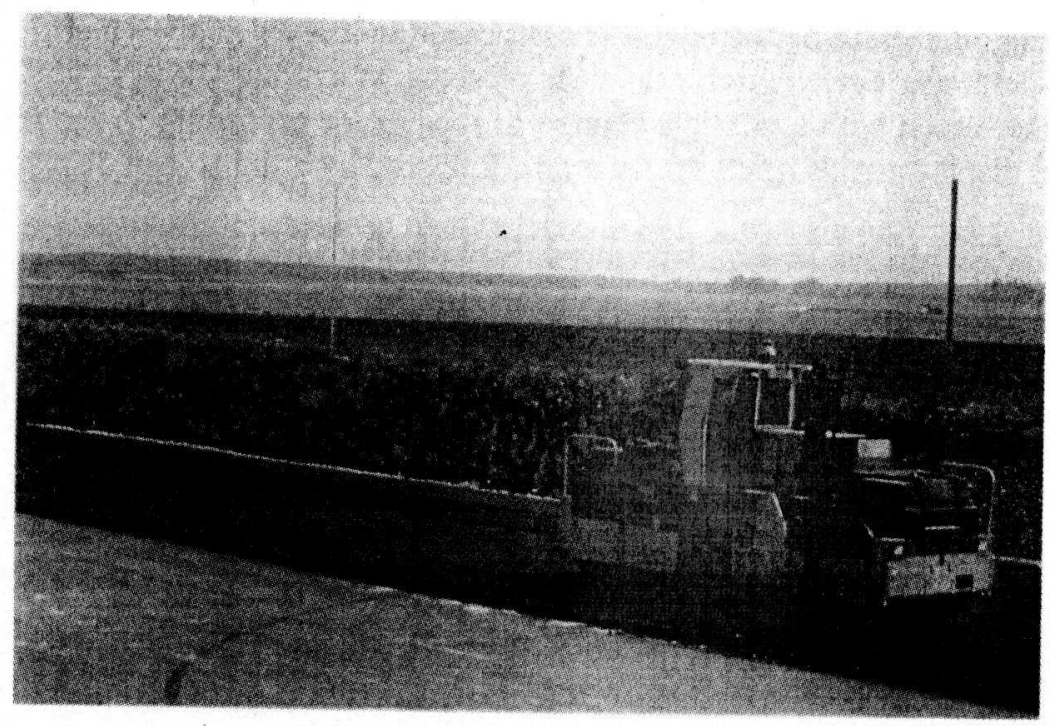

Figure 5: road construction site experimentation

The major problem encountered when placing the prototype equipment on board of the compactor, was to find the best site for the man-machine interface because of the smallness of the cab. It is an important detail since the screen must be quickly within the reach of the operator without being in his way. Moreover, the screen must not mask the visibility of the operator. The time necessary for the operator to be initiated into the utilization of the system is very short, about one hour, and can be explained by the simplicity of use and by the fact that the information provided are directly translated through the cartography into notions well known by the operator (cross and longitudinal profiles, axises of the compaction strips, number of passes).

This experimental site gave us the opportunity to survey the functioning of the equipment while offering the driver an operating aid and studying his reactions.

The study was carried out on a total linear length of $900 \mathrm{~m}$ broken into 9 sections of $100 \mathrm{~m}$. The division in discrete sections was guided by the implantation necessities of the CAPSY ${ }^{\mathrm{TM}}$ beacons. So as to determine the position of the compactor with good accuracy, and 
according to the working conditions (presence of several site vehicles in the studied area. transverse and longitudinal cross-fall), 8 beacons must be placed for a linear length of $100 \mathrm{~m}$. Figure 6 shows the site of the dashboard in the compactor cab.

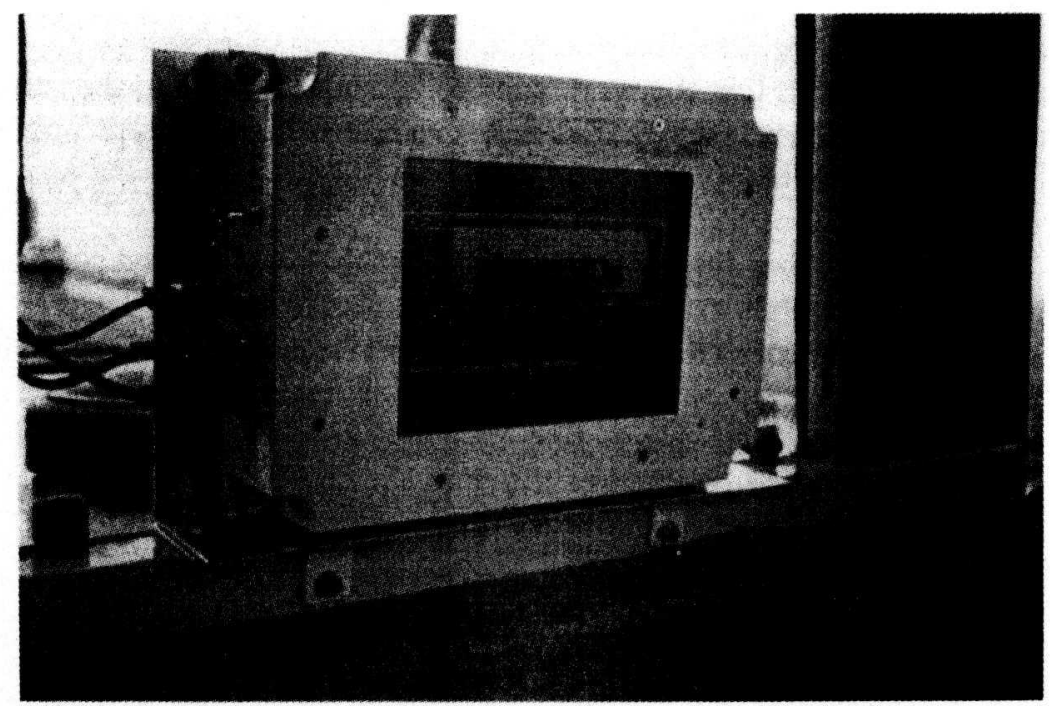

Figure 6: Man-machine interface placed on board of the compactor

In addition to real-time cartography, the on-board operating aiding software allows to display (when the compactor is stopped) the same information for the whole work site. Figure 7 illustrates this function by showing the outcome of the compaction work when one area is finished. Here the experimental compactor has a 6-m large pavement to compact from the axis of the project. By referring to this representation and using the arrows at the bottom of the screen, the operator is able to move virtually over the whole linear length of the work site and, thus, to control the work achieved very quickly.

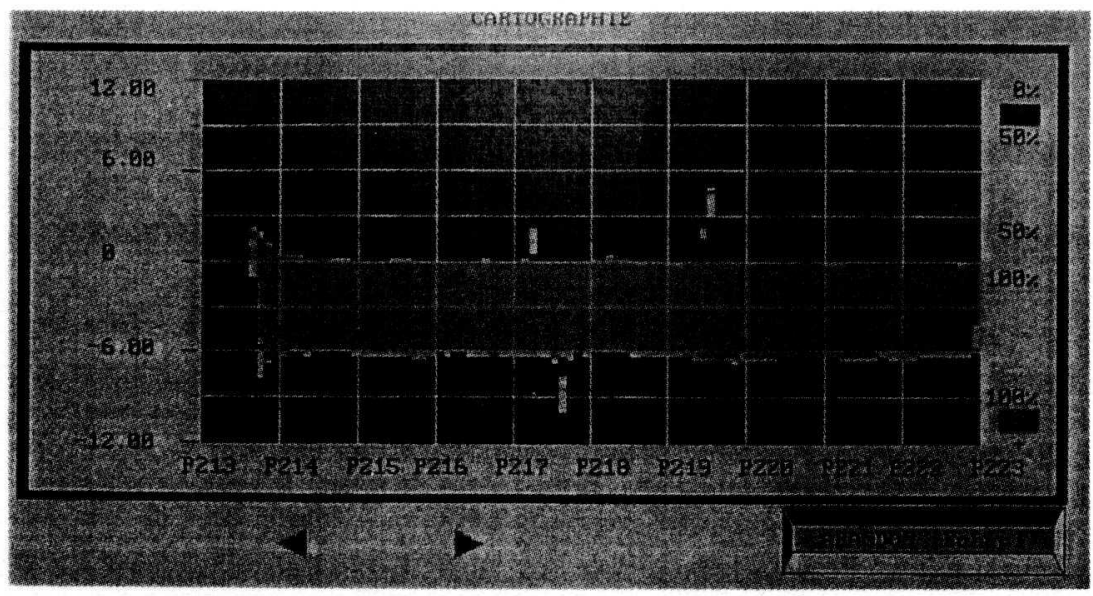

Figure 7: Cartography of an area after compaction 


\section{CONCLUSION}

The main conclusions we are able to draw from these first tests are:

- the operator adapts very easily to this new tool

- the operator is initiated very quickly into the system

- the operator fully trusts the system

- the operator feels release from counting the number of passes

- the system confirms that the work has been done according to the rule book

The experimental work site also allows us to consider the improvement observations about the MACC system of both the operator and the person responsible for the company :

- to reduce the bulk of the on-board computer

- to improve the embedding of the dashboard in the cab (site and size)

- to increase the legibility of the screen with regard to the surrounding lighting

- to couple the control of the compactor vibration function with the system so as to evaluate passes carried out only in the vibration mode.

- to replace the CAPSY ${ }^{\mathrm{TM}}$ positioning system, which, though a high-performance system, poorly fits the conditions of a road construction site (heavy implantation for the beacons, insufficient range for the laser, sensitivity to the rain and the vibrations).

- to display the cartography with a fourth level of color indicating over-compaction, a piece of information very important for the road builder (additional production).

All the lessons learned from this first experiment have contributed to the development of a new version of the MACC system. The main modification consists in the replacement of the CAPSY $^{\mathrm{TM}}$ sensor by a differential real-time GPS positioning system (Global Positioning System). This new system is more suitable for road construction sites and requires the implantation of only one reference station, which can be situated some kilometers away from the implementation site. The accuracy of the first measurements achieved with this system on our station for the study of localization systems, SESSYL [2], is centimetric for the three axises.

The following modifications have also been made:

- miniaturization of the on-board computer (standard PC104)

- coupling of the vibration control to the MACC system and evaluation of the passes carried out in the vibration mode

- new version of the man-machine interface (simplification of the controls)

- addition of a fourth level of color for the cartography

The tests of the new MACC system will start in March 1996, in collaboration with the same road construction company, on a large motorway construction site (A28). The test will be carried out on a linear length of $30 \mathrm{~km}$ with a single-cylinder compactor for the compaction of a pavement base.

[1] Field Applications with CAPSY

REFERENCES

Leon De Vos, TNO-BOUW, The Netherlands

Bob Hasara, Spectra Physics Laserplane, USA

10th IASRC, May 1993, Houston, USA

[2] SESSYL: The study of the positioning systems for road construction robotics François Peyret, Laboratoire Central des Ponts et Chaussées, Nantes, France 12th IS $\Delta$ RC, May 1005, Warcaw, Poland 